\title{
Chinese Medicine Characteristic Nursing for Mild and Ordinary Types of SARS-CoV-2 Virus Infected Patients
}

\author{
WU Meirong ${ }^{1 *}$ \\ ${ }^{1}$ Nanchong Traditional Chinese Medicine Hospital, Nanchong China
}

\begin{abstract}
SARS-CoV-2 is a new type of coronavirus, its source has not been accurately traced back. According to relevant medical studies, this noncellular morphological microorganism of about $60-140 \mathrm{~nm}$ in length may cause severe respiratory, cardiovascular and digestive system damage to infected patients. The disease caused by the virus is named as COVID-19 (Corona Virus Disease 2019) by WHO. For COVID-19, no matter it is an anonymous asymptomatic treatment by Chinese and Western medicine, there is currently no specific medicine. The Traditional Chinese Medicine (TCM) book named as "Basic Questions·Acupuncture Theory" points out that this kind of disease is a epidemic disease, easy to infect each other, and its symptoms are similar for all ages. Based on many years of traditional Chinese medicine nursing experience and authoritative literature, the author summarizes a set of traditional Chinese medicine characteristic nursing program for mild / ordinary type of SARS-CoV-2 virus infected patients, which can make the patient recover quickly. The main idea is to improve the patients' resistance to the coronavirus by using traditional Chinese medicine characteristic nursing such as diet conditioning, scraping, massage, plaster, baduanjin exercise, moxibustion and Chinese medicine emotion nursing on the basis of TCM syndrome differentiation and classification. The TCM characteristic nursing program summarized in this paper has been applied to patients with mild / ordinary type of new coronavirus pneumonia, and has achieved good nursing results.
\end{abstract}

\section{Introduction}

The COVID-19 with extremely infectivity and higher mortality rate has brought great disaster to all countries of the world. Of particular concern is that there is no specific drug for the coronavirus infected, and the vaccine that protects susceptible people has not yet completed phase 3 clinical trials. However, China has won a great victory in the prevention and treatment of this strong infectious disease. In this process, the TCM characteristic nursing has played a very important role. On the basis of syndrome differentiation and classification of TCM , combined with the author's nursing experience, the author summarizes several different types of characteristics nursing Programmes of TCM for mild / ordinary type of SARS-CoV-2 virus infected people.

\section{Etiology analysis and syndrome differentiation and classification of TCM}

"Basic Questions· Acupuncture Theory" said that "if the epidemics are coming, people are easily infected, no matter what age is, their symptoms are similar". It not only emphasizes that epidemic disease is easily infectious, but also emphasizes that epidemic disease has high similarity in clinical manifestation. The COVID-19 is epidemic and infectious, so the disease belongs to the category of "epidemic disease" of TCM[1]. The disease is caused by coronavirus, so it must be a invasive evil. From the TCM viewing, the disease is caused by the dampness, so it can belong to the category of dampness evil, which is called "dampness-toxicity-plague"[2-3]. According to different clinical manifestations, the disease can be classified as cold -dampness stagnated in the lung (mild), dampness-toxin stagnated in the lung (ordinary type), pestilence-toxicity blocking the lung (severe type), internal blockade and external collapse (critical type) and inefficiency of Lung and Spleen (Recovery Period).

\section{Characteristic nursing of TCM for cold -dampness stagnated in the lung}

\subsection{Typical symptoms of cold -dampness stagnated in the lung}

Cold-dampness stagnated in the lung syndrome refers to the cold and dampness is depressed in the lung, with fever, fatigue, pain around the body, cough, sputum, chest tight breath, anorexia, nausea, vomiting, stool sticky and greasy, tongue light fat teeth marks or pale red, fur white thick rot greasy or white greasy, pulse moistening or slippery as the main manifestation of the new coronavirus pneumonia mild syndrome[1]. 


\subsection{Diet conditioning}

Chinese medicine believes that the rise and fall of healthy trends determines the outcome and prognosis of the disease ,"healthy trends exist, evil can not make trouble", healthy trends depends on the stomach grain food for its nourishment, so in the diet should pay attention to the spleen and stomach. Avoid overeating, cold, bitter and acidic food [5]. Recommended beef and mutton, mushrooms, and aromatic vegetables such as scallions, garlic seedlings, Chinese toon, Artemisia annua, leek and other foods that contribute to hair growth. Second, the patients should drink more water, because drinking water has the role of "diarrhea lung ". The patients should drink boiled water, mild tea or raw coix seed water, to clear the dampness and heat. For patients with poor appetite and insufficient food intake, the main purpose should be "Reconciliate of healthy trends and blood which running on the body surface, Strengthen the spleen and kidney, strengthen the body's protective function"[1]. Eating nutritional fortified foods, formula foods or nutritional supplements for special medical uses, and micronutrients such as protein supplements, vitamin A,B,C,and D [6].

\subsection{Head scraping and acupoint massage}

Mild fever, headache, insomnia and other symptoms are more obvious, head scraping and acupoint massage can significantly alleviate the related symptoms of patients. Its operation essentials are: firstly the nurse uses 40 degrees warm water to wipe the patients' head and face, then chooses buffalo horn scraping board. Scraping is carried out from top to bottom, from front to back. The first line is facial scraping, starting from Yintang acupoint, to Zanzhu, Yuyao, Sizhukong to Taiyang, each side scraping 20 30 times, the acupoints have been shown in Fig.1. After wiping the head, combing the du pulse, left and right bladder meridians, from the forehead to the back side of head 10 times each; then wiping the head on both sides, from the forehead to the back, 20 30 times each side. Then Baihui $\mathrm{Yu}$ as the center, radiate to the whole head ,20 30 times on both sides; finally point the Taiyang, Fengchi, Fengfu 10 times each[7].

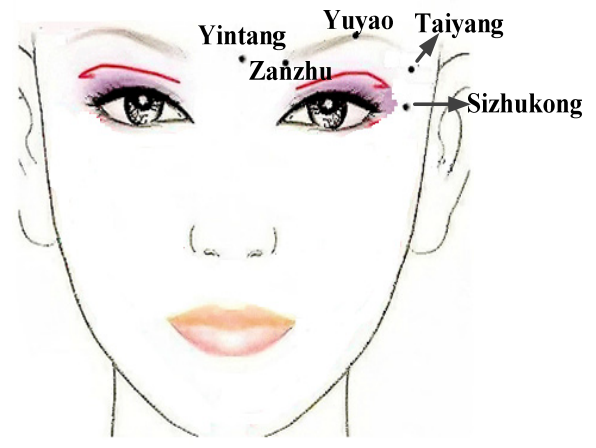

Fig1. Facial scraping acupoints

\subsection{Warming spleen and relieving diarrhea acupoint plaster}

Mild patients may also have diarrhea, warm spleen diarrhea acupoint plaster treat the patients caused by
SARS-CoV-2 virus total efficiency as high as $94.1 \%$, and no any toxic side effects [8]. Acupoint plaster not only belongs to internal disease with external treatment, but also belongs to traditional Chinese medicine characteristic nursing. According to information provided by Benifitel Pharmaceutical Company, Lishui City, Zhejiang Province, the warm spleen anti-diarrhea acupoint plaster is composed of cinnamon $10 \mathrm{~g}$, clove $6 \mathrm{~g}$, radix aucklandiae $6 \mathrm{~g}$, fennel $5 \mathrm{~g}$, nutmeg $5 \mathrm{~g}$, pepper $3 \mathrm{~g}$. The nurse mixed the medicine and ground it into fine powder, added sterile water and mixed it into paste, scooped it on the gauze with a spoon, and then applied it to the navel, Zusanli and Sanyin Interchange. Three hours per day. A course of treatment is 10 days. Note: Fasting seafood, spicy and irritating food should be forbade during treatment, waterproof during treatment, observe whether the skin is red and damaged, if any, remove it as soon as possible.

\subsection{Baduanjin}

Baduanjin is a COVID-19 rehabilitation exercise method recommended by the Chinese Rehabilitation Society. It can exercise and regulate the respiratory function, cardiopulmonary function and digestive function of mild patients. The first and last two types of "Baduanjin" pay attention to the regulation of the whole body, while the middle six paragraphs focus on all parts of the body, such as "two hands to support the sky in three Jiao ", two arms can stretch limbs, deep breath can improve respiratory function. "Pull the bow from left to right like shooting eagle", chest expansion and arm extension can enhance cardiopulmonary function;"regulate spleen and stomach by lifting single arm", by stretching visceral organs people can promote intestinal peristalsis and improve digestive function [9-10]. First, the nursing staff who passed the professional examination of Baduanjin explained the technical essentials to the patients, and then exercised for about 30 minutes a day, taking 10 days as a course of treatment. Exercise until the sixth day, the symptoms of muscle soreness, fatigue basically disappeared, lung function basically returned to normal, gastrointestinal digestion and sleep basically returned to normal [11].

\section{Characteristic nursing of TCM for dampness-toxin stagnated in the lung}

Special nursing for mild diseases such as diet nursing, head scraping and massage, warm spleen and diarrhea acupoint plaster is also suitable for ordinary patients. Baduan Jin can also exercise under the guidance of nurses in the case of good mental state and energy. However, because the common type patients are slightly more serious than the mild patients, it is necessary to formulate a differential nursing program of TCM.

\subsection{Typical symptoms of dampness-toxin stagnated in the lung}

The typical manifestations of new coronavirus pneumonia dampness and lung syndrome are fever, cough and phlegm, yellow phlegm, shortness of breath, abdominal 
distension and constipation. Dark red tongue, fat tongue, yellow fur greasy or yellow dry [4].

\subsection{Moxibustion}

Folium artemisiae argyi has effects of anti-allergic, antibacterial, anti-virus and enhance immunity[12]. The aromatic oil and essential oil can regulate the body by burning and ironing at specific acupoints or parts of the body surface. The photo-thermal radiation can directly stimulate the local tissue receptor to achieve the effect of warming the meridian, regulating healthy trends and blood, and moving cold and dampness. According to the guidance of acupuncture and moxibustion intervention of new coronavirus pneumonia (first edition) of the Chinese Acupuncture and moxibustion Society, four acupoints of Hegu, Taichong, Zusanli and Guanyuan were selected for dampness-toxic stagnation lung syndrome. Hegu and Taichong are called Siguan acupoints, which have the effect of dispelling wind and dispersing cold, relieving depression and relieving pain, calming the heart and tranquilizing the mind. Every afternoon or before dinner, nursing staff slowly cauterizate the acupoints of Hegu, Taichong, Zusanli, Guan Yuan. 40 min each time once a day [14].

\subsection{Shou-Tai-Yin lung meridian massage}

The symptoms of cough and shortness of breath may occur in patients with dampness-toxin stagnated in the lung, so it is necessary to monitor the vital signs, the degree of short wheezing, the duration, the oxygen saturation, and evaluate the degree of hypoxia. According to the degree of dyspnea and accompanying symptoms, nursing staff massage the patients with Shou-Tai-Yin lung meridian. The main acupoints of operation are as follows: starting with the epigastric part of Zhongiiao, connecting the intestine downward. The meridian acupoints are Zhongfu, Yunmen, Tianfu, Xia Bai, Chize, Kongzui, Jingqu, Taiyuan, Yuji and Shaoshang. The figure 2 shows the Shou-Tai-Yin lung meridian. Massage once a day, each time about $20 \mathrm{~min}$, continuous massage for 7 days, the patients' cough, shortness of breath symptoms disappeared for the longest 6 days, the shortest 2.5 days, the average (4.2 \pm 0.4$)$ days, the patients' satisfaction are $100.0 \%[15]$.

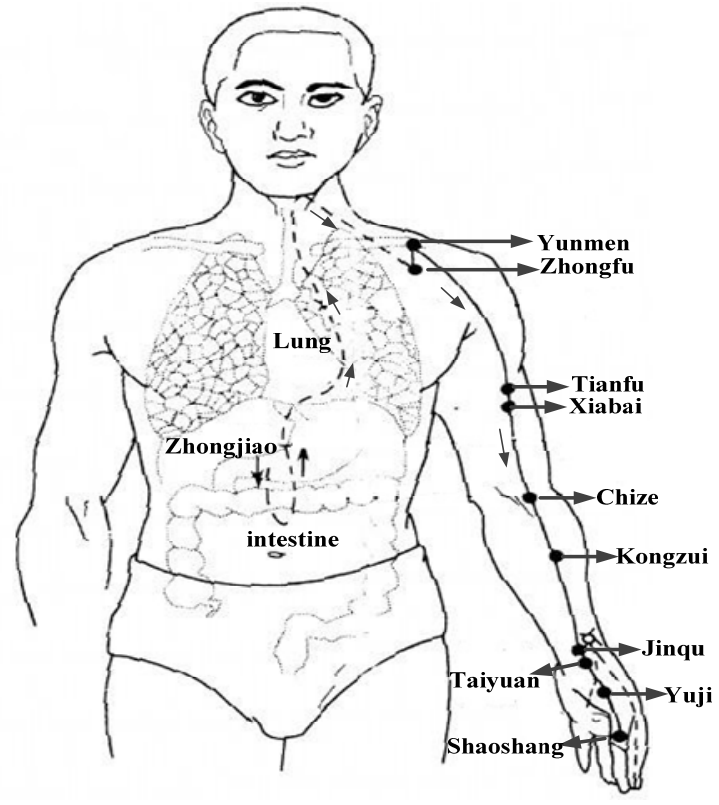

Fig2. Shou-Tai-Yin Lung meridian

\subsection{Emotional Care in TCM}

The psychological pressure of patients with dampnesstoxin stagnated in the lung may be much greater than that of mild patients, so they need emotional care. Five-tone therapy is based on the five-tone theory recorded in the Yellow Emperor's Internal Classic, the use of "Do Re Mi Sol La" different tone of music to treat diseases, especially for emotional diseases. The effect of physical and mental symptoms or crisis events on individuals can also be visualized as a specific object (symbol), and the target symptoms or emotions can be dealt with by concrete carriers. Finally, the effect of eliminating psychological pressure is achieved. The specific operation steps are as follows :(1) three adjustments and relaxation;(2) determination of target symptoms ;(3) guiding symbolism ;(4) unified carrying material as disinfection apparatus related to epidemic situation;(5) emptying. About 30 min per operation[17-18].

\section{Summary}

According to the mild type of SARS-CoV-2 virus infected (TCM type is cold -dampness stagnated in the lung), the characteristic nursing scheme of TCM is diet nursing, scraping and acupoint massage, warming spleen and stopping diarrhea acupoint plaster and Baduanjin, and the characteristic nursing scheme of TCM for ordinary type (TCM type isdampness-toxin stagnated in the lung) is to increase moxibustion, Taiyin lung meridian massage and Chinese medicine emotion nursing on the basis of TCM nursing for mild patients. After verification by relevant literature, the above scheme has a very positive effect on mild and ordinary SARS-CoV-2 virus infected patients, which is worth to be promoted. 


\section{ACKNOWLEDGMENTS}

The author would thank the sponsor provided by the Research Project of Sichuan Education Department " Research on Pension Health Service for Retired People in Colleges and Universities " [Grant number:17SB0535]

\section{About the Author}

WU Meirong, female, 1982.08, graduated from North Sichuan Medical College, majored in nursing. She is a supervisor nurse ot the Nanchong Traditional Chinese Medicine Hospital, the main research field is traditional chinese medicine nursing.

\section{REFERENCES}

1. General Office of the State Health and Health Commission,2020-03-28. Office of the State Administration of traditional Chinese Medicine. Diagnosis and Treatment of New Coronavirus Infected Pneumonia (Trial 7th Edition); http //bgs.satcm.gov. cn /zhengcewenjian /2020-0328/12576.html.

2. Miao Qing, Cong Xiaodong, Wang Bing, et al, 2020, Traditional Chinese Medicine Understanding and thinking of New Coronavirus Infected Pneumonia. Chinese Medicine Journal ,,16(6):2.

3. Wang Yuguang, Qi Wensheng, Ma Jiaju, et al, 2020, Clinical Characteristics and Dialectical Treatment of New Coronavirus (2019- nCoV) Pneumonia. TCM Journal of Traditional Chinese Medicine, 281-285

4. Beijing Society of traditional Chinese Medicine Nursing Committee, 2020, Beijing new coronavirus pneumonia traditional Chinese medicine nursing program (trial). Chinese medicine in Beijing.39(5) 423-426.

5. Zhao Yulan, Wu Li Xian, 2011, Discussion on Dietary Nursing in TCM Theory. Bright Chinese Medicine ,26(2):386-387.

6. Zhong Yuan, Liu Shu Zhang Yuxin, et al, 2020, Key points of traditional Chinese medicine nursing prevention and control of new coronavirus pneumonia. Chinese nursing education.17(4): 311315.

7. Guo Jiaying, Wang Qiaozhi, ou Xiujuan, et al, 2018, Clinical study on the treatment of exogenous febrile diseases with head scraping. New Chinese Medicine ,9(50):212-214.

8. Lai Junmei, Zheng Yongfei, Hu Chunyan, et al, 2020, Effect of Warming spleen and relieving diarrhea acupoint plaster on Anti-Viral Diarrhea of New Crownavirus Pneumonia:. Observation Zhejiang Journal of Integrated Chinese and Western Medicine 30(4):268-270.

9. Zhang Qi, Jin Hongbin, Fan Tongshun, et al, 2018, Fitness Baduanjin Exercise for College Students' Chronic Neck Efficacy of pain patients. Chinese
Journal of physical Medicine and Rehabilitation 40(8):625-627.

10. Yu Yiyong, 2019, Evaluation of the efficacy of fitness Baduanjin in pulmonary rehabilitation in stable stage of chronic obstructive pulmonary disease. World's latest Medical Information Abstracts 19(84):210-211.

11. Zhang Jing, 2020, Experience of Traditional Chinese Medicine Characteristic Nursing on COVID-19 with Cold-dampness Stagnated Lung Syndrome for One Case.in China Chinese Traditional Chinese Medicine Modern Distance Education, No .2020,18(5): X4-X6

12. Liang Kunlun, Sun Jinhao, Zhang Ruifang, 2015, Study on Biochemical Composition and Pharmacological Action of Artemisia argyi, 2015(30):80-81.

13. Chinese Acupuncture Society, 2020, Guidance on Acupuncture and moxibustion Intervention for New Coronavirus Pneumonia of Chinese Acupuncture Society Acupuncture and moxibustion in China (First Edition) ,40(2):111.

14. Chen Xia, Huang Wei, Liu Baoyan, et al, 2020, Moxibustion therapy in prevention and treatment of coronavirus disease 2019 (COVID-19): construction and application of non-contact diagnosis and treatment model. Chinese Acupuncture \&Moxibustion, 1-7

15. Wei Guangrong, 2020, To explore the effect of traditional Chinese medicine nursing on relieving cough and shortness of breath. Nursing Research, 2020(12):153-154.

16. Dong Renqi, Zhou Xia, Li Li, 2019, Advances in the treatment of post-stroke depressionstroke Depression, 27(10):109-111.

17. Zhao Xudong, Liu Zhongmin. 2020, Anti-epidemic peace of mind-the-epidemic psychological self-care rescue of the whole people. Shanghai: Shanghai Science and Technology Press ,110

18. Zhou Xia, Liu Tianjun, Li Li, 2020, Construction and Application of the Intervention Mode of Chinese Medicine's Characteristic Psychological Crisis under the New Coronary Virus Pneumonia. 28(3): 9-13. 